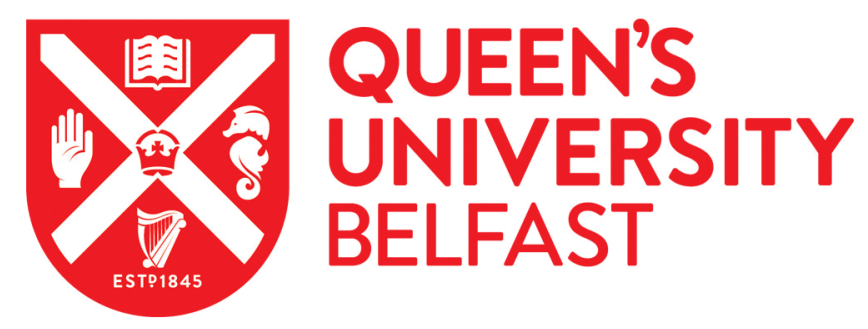

\title{
Sequential injection of domain walls into ferroelectrics at different bias voltages: Paving the way for "domain wall memristors"
}

\author{
Whyte, J., McQuaid, R., Ashcroft, C. M., Einsle, J., Canalias, C., Gruverman, A., \& Gregg, M. (2014). Sequential \\ injection of domain walls into ferroelectrics at different bias voltages: Paving the way for "domain wall \\ memristors". Journal of Applied Physics, 116, [066813]. https://doi.org/10.1063/1.4891347
}

Published in:

Journal of Applied Physics

Document Version:

Publisher's PDF, also known as Version of record

Queen's University Belfast - Research Portal:

Link to publication record in Queen's University Belfast Research Portal

\author{
Publisher rights \\ Copyright (year) American Institute of Physics. This article may be downloaded for personal use only. Any other use requires prior \\ permission of the author and the American Institute of Physics. \\ The following article appeared in Journal of Applied Physics and may be found at \\ http://scitation.aip.org/content/aip/journal/jap/116/6/10.1063/1.4891347 \\ Copyright 2014 J. R. Whyte, R. G. P. McQuaid, C. M. Ashcroft, J. F. Einsle, C. Canalias, A. Gruverman and J. M. Gregg \\ This article is distributed under a Creative Commons Attribution 3.0 Unported License.
}

\section{General rights}

Copyright for the publications made accessible via the Queen's University Belfast Research Portal is retained by the author(s) and / or other copyright owners and it is a condition of accessing these publications that users recognise and abide by the legal requirements associated with these rights.

Take down policy

The Research Portal is Queen's institutional repository that provides access to Queen's research output. Every effort has been made to ensure that content in the Research Portal does not infringe any person's rights, or applicable UK laws. If you discover content in the Research Portal that you believe breaches copyright or violates any law, please contact openaccess@qub.ac.uk. 


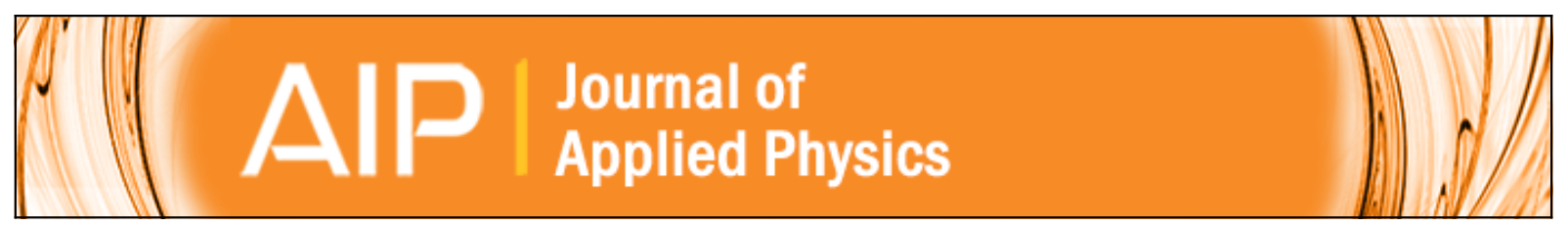

\section{Sequential injection of domain walls into ferroelectrics at different bias voltages:}

\section{Paving the way for "domain wall memristors"}

J. R. Whyte, R. G. P. McQuaid, C. M. Ashcroft, J. F. Einsle, C. Canalias, A. Gruverman, and J. M. Gregg

Citation: Journal of Applied Physics 116, 066813 (2014); doi: 10.1063/1.4891347

View online: http://dx.doi.org/10.1063/1.4891347

View Table of Contents: http://scitation.aip.org/content/aip/journal/jap/116/6?ver=pdfcov

Published by the AIP Publishing

\section{Articles you may be interested in}

Nucleation and growth of periodic domains during electric field poling in flux-grown K Ti O P O 4 observed by atomic force microscopy

Appl. Phys. Lett. 88, 032905 (2006); 10.1063/1.2166678

High-resolution domain imaging on the nonpolar y-face of periodically poled KTiOPO 4 by means of atomic force microscopy

Appl. Phys. Lett. 83, 734 (2003); 10.1063/1.1593834

Nanoscale imaging of domains and domain walls in periodically poled ferroelectrics using atomic force microscopy

Appl. Phys. Lett. 80, 1622 (2002); 10.1063/1.1455700

Bulk ferroelectric domain nucleation in KTiOPO 4 crystals

Appl. Phys. Lett. 79, 2964 (2001); 10.1063/1.1413959

Anisotropic domain structure of KTiOPO 4 crystals

J. Appl. Phys. 90, 1950 (2001); 10.1063/1.1385575

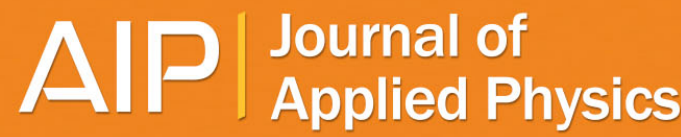

Journal of Applied Physics is pleased to announce André Anders as its new Editor-in-Chief 


\title{
Sequential injection of domain walls into ferroelectrics at different bias voltages: Paving the way for "domain wall memristors"
}

\author{
J. R. Whyte, ${ }^{1}$ R. G. P. McQuaid, ${ }^{1}$ C. M. Ashcroft, ${ }^{1,2}$ J. F. Einsle, ${ }^{1}$ C. Canalias, ${ }^{3}$ \\ A. Gruverman, ${ }^{4}$ and J. M. Gregg ${ }^{1, a)}$ \\ ${ }^{1}$ Centre for Nanostructured Media (CNM), School of Maths and Physics, Queen's University Belfast, \\ University Road, Belfast BT7 1NN, United Kingdom \\ ${ }^{2}$ Department of Physics, Cavendish Laboratory, J. J. Thomson Avenue, Cambridge CB3 OHE, United Kingdom \\ ${ }^{3}$ Department of Applied Physics, Royal Institute of Technology, Roslagstullsbacken 21, 10691 Stockholm, \\ Sweden \\ ${ }^{4}$ Department of Physics and Astronomy, University of Nebraska Lincoln, Nebraska 68588-0299, USA
}

(Received 27 January 2014; accepted 21 March 2014; published online 11 August 2014)

\begin{abstract}
Simple meso-scale capacitor structures have been made by incorporating thin $(\sim 300 \mathrm{~nm})$ single crystal lamellae of $\mathrm{KTiOPO}_{4}$ (KTP) between two coplanar Pt electrodes. The influence that either patterned protrusions in the electrodes or focused ion beam milled holes in the KTP have on the nucleation of reverse domains during switching was mapped using piezoresponse force microscopy imaging. The objective was to assess whether or not variations in the magnitude of field enhancement at localised "hot-spots," caused by such patterning, could be used to both control the exact locations and bias voltages at which nucleation events occurred. It was found that both the patterning of electrodes and the milling of various hole geometries into the KTP could allow controlled sequential injection of domain wall pairs at different bias voltages; this capability could have implications for the design and operation of domain wall electronic devices, such as memristors, in the future. (C) 2014 AIP Publishing LLC. [http://dx.doi.org/10.1063/1.4891347]
\end{abstract}

\section{INTRODUCTION}

In 1971, Chua $^{1}$ stated that the three known basic two terminal components in electrical circuits (resistors, capacitors, and inductors) were insufficient to fully describe the interrelationships between all possible pairs of the four fundamental circuit variables: current $(I)$, charge $(q)$, voltage $(V)$, and flux-linkage $(\phi)$. He postulated that one further two terminal component must exist in order to link together flux and charge $(d \varphi=M d q) .{ }^{1,2}$ He termed this new fundamental circuit element the "memristor" (abbreviation of "memory resistor"). At that time, no such component was known and it was not until 2008 that Strukov et al. ${ }^{2}$ and Yang et al. ${ }^{3}$ claimed the first incarnation of real memristor devices (in metal/oxide/metal structures $\left.\left(\mathrm{Pt} / \mathrm{TiO}_{2} / \mathrm{Pt}\right)\right)$. As noted by Mathur, ${ }^{4}$ however, the magnetoelectric coupling coefficient in multiferroics should be inversely proportional to $\frac{d \varphi}{d q}$ and therefore memristors could have been previously made within the magnetoelectric multiferroic community and overlooked. In any event, since 2008, the creation and investigation of memristors has become a rather active area for research, fuelled by potential use in neuromorphic computer architecture (due to the similarity between memristors and biological synapses $)^{2,5-7}$ as well as other more conventional logic and memory applications. ${ }^{8}$

As part of this ongoing research, ferroelectric materials have been recently championed for their memristive properties: ${ }^{5,7,9-13}$ very large differences in the resistance of ferroelectric tunnel junctions (FTJ's), with resistance ratios of up

\footnotetext{
a) Author to whom correspondence should be addressed. Electronic mail: m.gregg@qub.ac.uk
}

to $10^{4}$, have been seen when the sense of dipole polarization is reversed. 5,7,9-11,13,14 Polarization-controlled binary memory and ON/OFF transistor states are clearly implied. Chanthbouala et al. ${ }^{5}$ have demonstrated that, by controlling the exact mixed populations of domains with different orientations in FTJ's, large ranges of distinct resistance states can be accessed. Thus memristive behavior resulted from control of domain microstructure.

This is not, however, the only way in which the engineering of ferroelectric domains might allow for memristor development: another approach exploits the recently discovered phenomenon of enhanced conductivity along certain types of domain walls in ferroelectric and multiferroic oxides. ${ }^{15-30}$ The realization that domain walls can act as conducting channels has prompted a number of resistancerelated devices to be proposed, where operation is solely based on the creation or annihilation of conducting domain walls that traverse interelectrode-gaps. If control over the number of high conductivity domain walls connecting electrodes could be developed, then a series of distinct resistance states could be created, and thus a tunable memristor realized. This would be an exciting development in the embryonic field of "domain wall electronics."

A key issue is in finding a way to reliably control both the locations and applied bias level at which successive sets of domain walls can be injected between electrode pairs in a ferroelectric capacitor device. In this article, we explore how patterning of both electrodes and ferroelectrics can introduce local "hot-spots" in the electric field distribution, where the field intensity at different "hot-spots" can be varied. Injection of reverse domains at a critical field value needed for nucleation was expected to occur at different bias 
voltages for each hot-spot: when the bias was sufficient to cause each hot-spot field intensity to be equal to or greater than the critical nucleation field, domain wall injection events were expected. Using this strategy, the sequential injection of domain wall pairs at specific locations and applied bias voltages was achieved, although not always completely as expected.

\section{EXPERIMENTAL METHOD}

All observations were made on $(010)_{\text {orthorhombic (o) la- }}$ mellar slices of uniaxial ferroelectric $\mathrm{KTiOPO}_{4}$ (KTP) ( $\sim 300 \mathrm{~nm}$ in thickness), cut from a $(001)_{\mathrm{o}}$ oriented periodically poled bulk single-crystal, using Focused Ion Beam Milling (FIB) (either a FEI200TEM or a FEI Nova 600i DualBeam system).

Capacitors, with asperities patterned into the electrodes, introduced field hot-spots of the kind shown by finite element analysis (FEA) models in Fig. 1(a). The KTP lamellae were placed onto $\mathrm{MgO}$ single crystal substrates and both lamellae and substrates were sputter-coated with a thin film of platinum. The upgraded ion column optics of the Nova 600i facilitates using the $5 \mathrm{kV}$ ion beam to accurately and selectively pattern the Pt film on top of the KTP defining the interelectrode gap. Using a higher acceleration voltage, further patterning of the Pt film defines distinct and electrically isolated contact pads on the substrate. The sample was then annealed in air at $300^{\circ} \mathrm{C}$ for $6 \mathrm{~h}$ to expel gallium associated with ion implantation and contamination during milling, ${ }^{31}$ while minimizing the chemical alterations to the KTP. ${ }^{32}$ The sample was then briefly exposed to an acid etch $(3 \mathrm{M} \mathrm{HCl})$ to leave a pristine surface ready for piezoresponse force microscopy (PFM) imaging.

For capacitors with holes milled into the KTP within the interelectrode gap, samples were created using the exact procedure and setup detailed in Ref. 33. A platinized $\mathrm{MgO}$ substrate was used to create co-planar electrodes by FIB milling, leaving a $\sim 2 \mu \mathrm{m}$ wide interelectrode gap. The lamella was then placed across the gap before FIB-milling holes of different geometries (on a FEI Nova 600i DualBeam system). A thermal anneal (at $400{ }^{\circ} \mathrm{C}$ for $6 \mathrm{~h}$ ) was then used to expel gallium ions and the sample was acid etched as before. Then platinum was applied to the lamella edges parallel to the interelectrode gap, using electron-beam-induced-deposition on the DualBeam system to secure the lamella in position.

PFM was performed using a Veeco Dimension 3100 AFM system with a Nanoscope IIIa controller and an EG\&G 7265 lock-in-amplifier. Planar switching was achieved by DC biasing the coplanar electrodes, generating an electric field parallel to the $(001)_{\mathrm{o}} \mathrm{KTP}$ lamella polarization axis.

\section{RESULTS AND DISCUSSION}

\section{A. Controlled domain wall injection using patterned electrodes}

Fig. 1 illustrates the expectations associated with switching capacitor structures with patterned electrodes, described above. From the results of the Quickfield FEA modeling presented in Fig. 1(a), it is clear that variations in the forms of the triangular asperities on the electrode lead to variations in the levels and spatial extent of local field enhancement when a potential difference is applied. Sharper triangular asperities, which extend farther into the interelectrode gap, generate field hot-spots of higher intensity and greater spatial extent than those associated with the broader triangular asperities, which do not protrude as far. As illustrated schematically in Fig. 1(b), field modeling should imply a distinct sequence of nucleation events during switching from a fully polarized state, leading to three distinct resistance states if the studied system exhibited significant domain wall conductivity enhancement (Fig. 1(c)). In such a case, the sequential nucleation of reverse domains and associated injection of discrete domain wall pairs should lead to a 3-state memristor.

Measurements on real coplanar devices (Fig. 2) largely mirror the schematic expectations of Fig. 1. Initially, the (a)

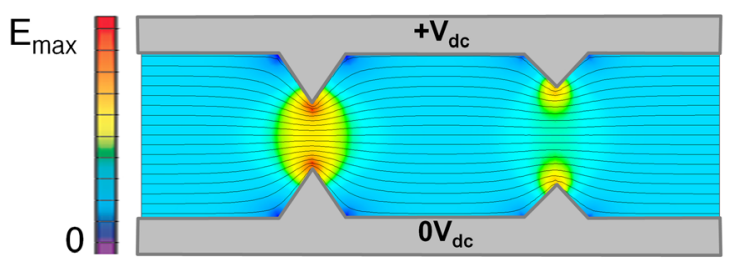

(c)

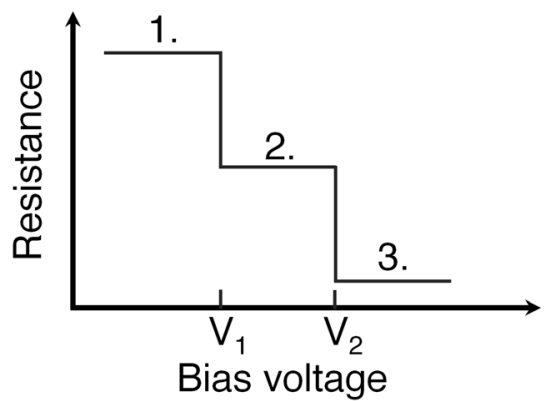

(b)

1. Initial state

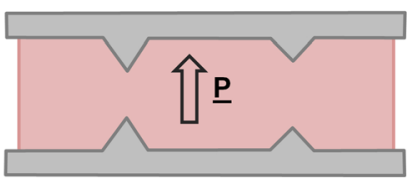

2. $\mathrm{V}_{1}>\mathrm{V}_{\text {crit }}$

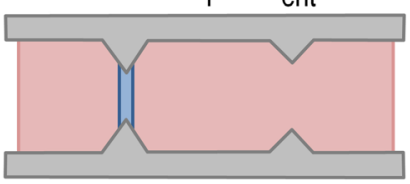

3. $\mathrm{V}_{2}>\mathrm{V}_{1}$

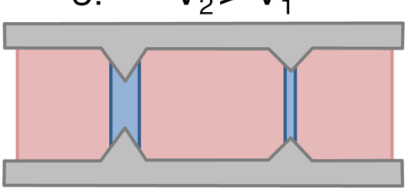

FIG. 1. (a) An electric field model for a KTP capacitor with notched electrodes where the left set of asperities are sharper than the right and the local electrode gap between opposing apices is closer. Color scale bar indicates electric field intensity. (b) (i) Schematic illustration showing the initial mono-domain state of a ferroelectric capacitor, (ii) after delivery of a voltage pulse $\left(\mathrm{V}_{1}\right)$ that generates a local electric field higher than the critical switching field at the left asperity but not for the right one. Only one pair of conducting domain walls is therefore injected. (iii) At a threshold higher voltage pulse $\left(\mathrm{V}_{2}>\mathrm{V}_{1}\right)$, a second pair of domain walls is injected at the right asperity. (c) Schematic graph illustrating different resistance states that depend on the voltage-controlled domain wall density. 
(a)

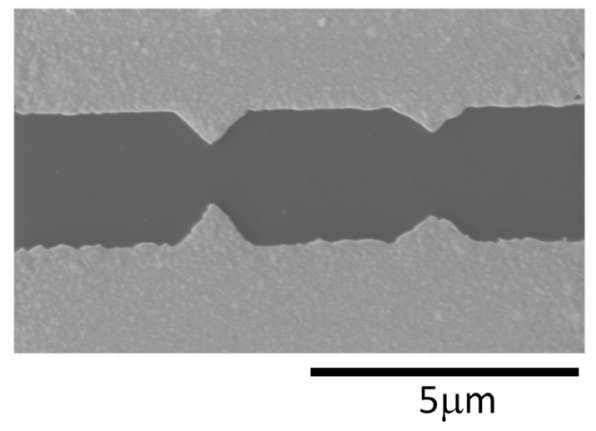

(c)

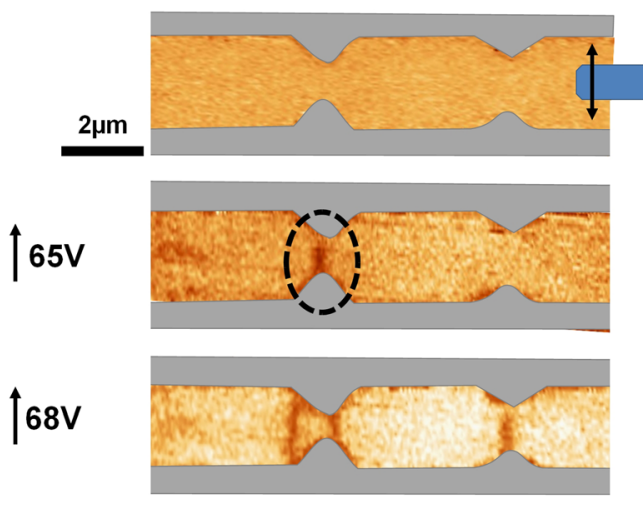

(b)

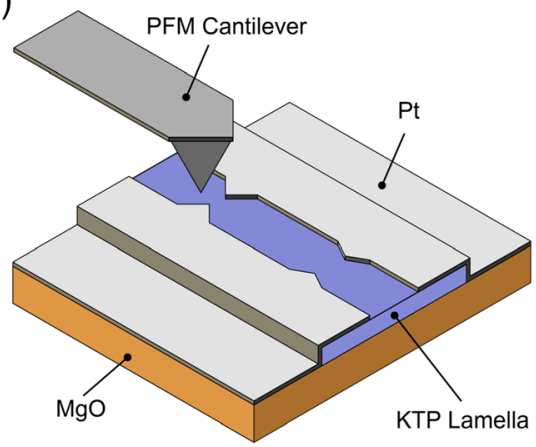

LPFM phase:
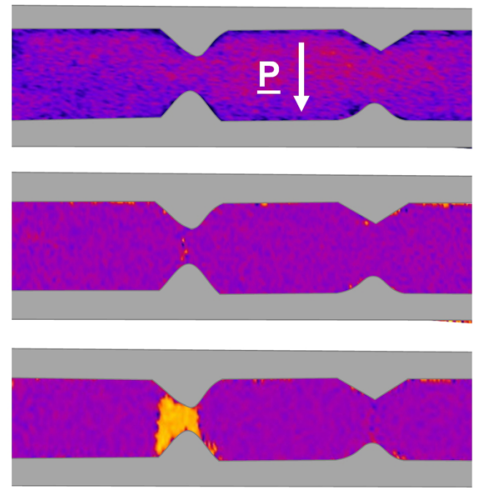

FIG. 2. (a) SEM image of notched electrodes on platinum sputtered KTP lamella created via FIB milling. (b) A schematic of the experimental setup for PFM investigations. (c) The lateralPFM amplitude and phase images showing the initial mono-domain state, one pair of injected domain walls after application of a $65 \mathrm{~V} 100 \mu \mathrm{s}$ pulse then, after being reset back to a monodomain state, two pairs of injected domain walls after a $68 \mathrm{~V} 100 \mu$ s pulse. capacitor was fully poled such that all polarization in the interelectrode gap was in one direction, indicated by both the uniform amplitude image (top left panel in Fig. 2(c)) and single phase in the lateral PFM data (top right panel in Fig. 2(c)). After an application of a $65 \mathrm{~V} 100 \mu$ s pulse in the opposite direction to that of the initial poling field, PFM images revealed a pair of domain walls injected at the points of the most significant electrode asperities and the strongest expected local field enhancements (middle panels in Fig. 2(c)). After resetting the lamella back to its initial fully poled state, a larger $68 \mathrm{~V} 100 \mu$ s pulse was then applied. This resulted in two distinct nucleation events, one at each set of electrode asperities. From the relative width of the reverse domains, shown in the bottom panels in Fig. 2(c), it is clear that the domain nucleated at the region of strongest expected local field has grown larger than that at the weaker hot-spot. Nevertheless, this $68 \mathrm{~V}$ pulse has resulted in a state in which two pairs of domain walls traverse the interelectrode gap.

Such control over domain switching behavior is obviously very similar to that depicted schematically in Fig. 1(c), and thus patterning of the electrodes to create field hot-spots of different intensities looks to be a viable way in which multiple resistance states (Fig. 1(b)), based on discrete biascontrolled injection of different numbers of domain wall pairs, might be realized.

\section{B. Controlled domain wall injection using milled holes in KTP lamella}

In previous work, Whyte et al. ${ }^{33}$ demonstrated that lowpermittivity air holes, milled into a ferroelectric of higher permittivity, could create local regions of both field enhancement and denudation. Regions of field enhancement were previously found to act as nucleation sites during switching, while low field regions could cause domain wall pinning. Field variations could be used to modulate domain wall speeds during switching.

Building upon the domain nucleation control already demonstrated, ${ }^{33}$ the influence of the hole shape on the relative intensity of localized field hot-spots was investigated further using FEA modeling (note that in this set of experiments simple planar electrodes are used, i.e., no patterned asperities in electrode profile). The shape of an ellipse and triangle, for example, appear to yield quite different levels of field enhancements, shown pictorially in the Quickfield models in Fig. 3(a). The local field is enhanced, above that of the uniform field in the unpatterned regions of the dielectric, by $80 \%$ at the right vertex of the triangle, $47 \%$ at the left edge of the triangle and $30 \%$ at the oval edges. Thus, differential nucleation of domains at the various hot-spots might be expected at different levels of applied bias at each of the geometric features sequentially, in a similar manner to the previous approach using patterned electrodes.

The patterns modeled in Fig. 3(a) were milled into the interelectrode gap region of the KTP coplanar capacitor (Fig. 3(b)) and PFM was again used to examine the sites and bias levels at which reverse domains were nucleated. The lamella was once again poled into a monodomain state; the slow scan axis of the PFM was then disabled, so the same line (just below the patterned holes) was continually scanned, as indicated in the PFM amplitude image of the lamella in Fig. 4(a). Voltage pulses from $40 \mathrm{~V}$ to $50 \mathrm{~V}$ (in increments of $1 \mathrm{~V}$ ) were then applied in-situ while the PFM signal along this single line was continuously 
(a)

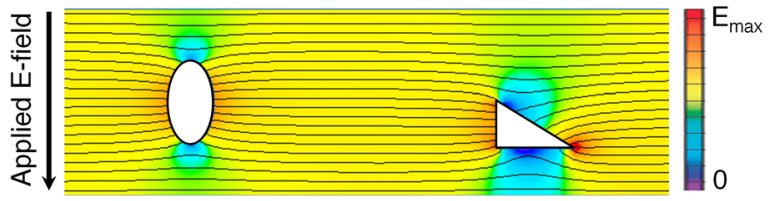

(b)

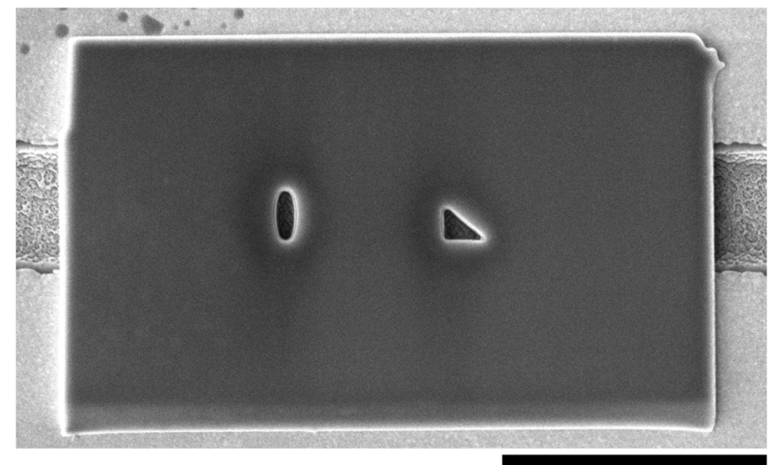

$5 \mu \mathrm{m}$

FIG. 3. (a) Electric field model of a KTP capacitor featuring elliptical and triangular air holes showing differing levels of field enhancement. Color scale bar indicates electric field intensity. (b) Top-down SEM image of KTP lamella with FIB milled holes placed across the interelectrode gap of the platinized $\mathrm{MgO}$ substrate.

monitored. Fig. 4(b) shows the change in average amplitude signal between the mono-domain state (no domain walls present) and the state after three specific voltage pulses (41, 48 , and $50 \mathrm{~V}$ ) had been applied. After $41 \mathrm{~V}$, we see the first domain wall pair injected on the inside edge of the triangle, characterized by the distinct dip in amplitude signal at that point. The next injection occurs at $48 \mathrm{~V}$ on the inside edge of the ellipse, before the final observed nucleation event at the outer vertex of the triangle (after the $50 \mathrm{~V}$ pulse). Afterwards, these locations and the presence of domain wall pairs were confirmed by a conventional PFM scan over the whole lamella, shown in Fig. 4(c).

While it is clear that domain walls have been injected at points of expected local field enhancement, the order in which they were induced (in terms of the relative levels of applied bias) was not as expected. The right vertex of the triangle should have generated the greatest levels of local field enhancement and hence nucleation of a reverse domain at the lowest bias relative to the other nucleation events; yet

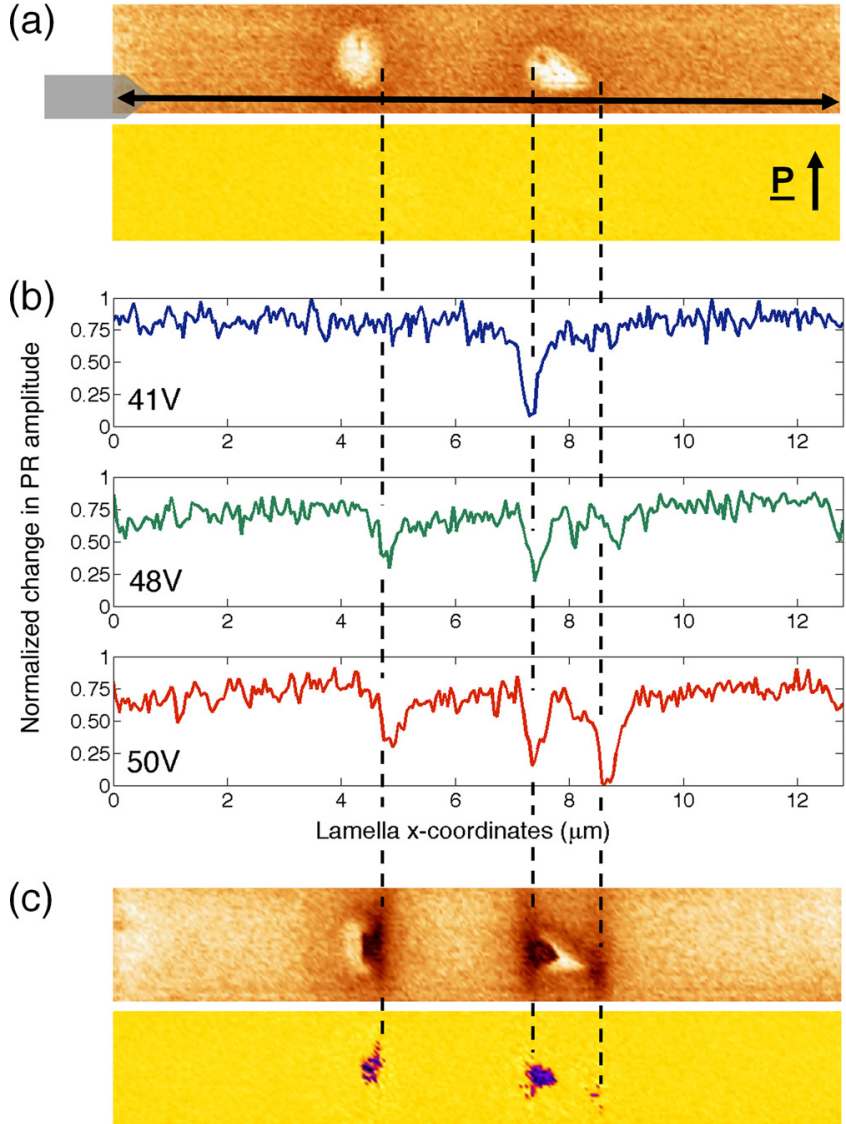

FIG. 4. (a) Lateral-PFM amplitude (top) and phase signal (bottom) of KTP lamella in fully poled mono-domain state. Cantilever schematic and black arrow illustrate the line that is continually scanned while voltage pulses are applied. (b) Normalized changes in the amplitude signal of the single scan line (from the original monodomain state at $0 \mathrm{~V}$ ) after application of $41 \mathrm{~V}$, $48 \mathrm{~V}$, and $50 \mathrm{~V}$ pulses, respectively. Prominent dips in piezoresponse (PR) amplitude indicate the appearance of a domain-wall pair. (c) Lateral-PFM amplitude (top) and phase (bottom) images showing the spatial arrangement of the injected domain walls across the whole interelectrode gap region. Black dashed lines act as a guide for the position of the hole edges.

definitive dips in domain wall amplitude at this location occurred at higher bias pulses than needed for nucleation events at the left edge of the triangle and right edge of the ellipse. In short, while the expected hot-spots accurately indicated the locations at which reverse domains would be (a)

(b)
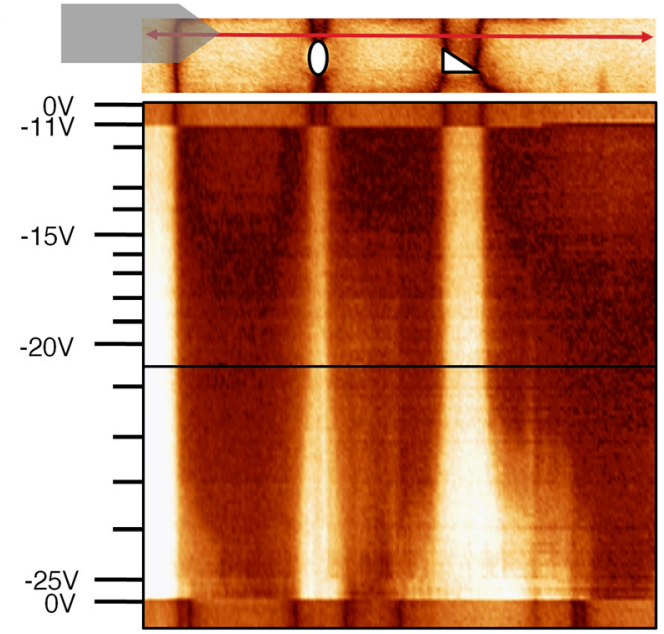

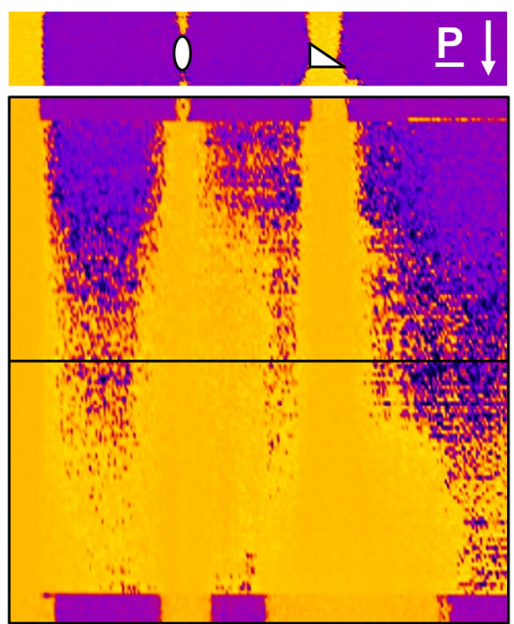

FIG. 5. Lateral-PFM amplitude (left) and phase (right) images of: (a) the remanent domain state across the interelectrode gap region of the KTP lamella after application of $+100 \mathrm{~V}$, showing domain walls residing along hole edges. (b) Time-lapse of a single scanline (along red arrow in (a)) where vertical axis indicates time elapsed increasing from top to bottom. A negative dc bias of increasing amplitude (from $-11 \mathrm{~V}$ to $-25 \mathrm{~V}$ ) is applied while observation of domain wall position is carried out in-situ. 
generated, the relative intensity of field enhancement as modeled was not an accurate guide as to the relative bias levels at which nucleation events would occur. It is possible that the FEA model may not have captured all of the relevant experimental factors contributing to electric field inhomogeneity. Nevertheless, the variations in milled hole geometry did cause pairs of domain walls to be injected at different locations at different levels of applied voltage. A memristive device exploiting this approach can be envisaged, even if operation was not quite as expected.

The effect that these holes had on the domain wall mobility during switching was also investigated. Here, the PFM slow scan axis was disabled with the tip positioned just above the holes, and an initial state with domain walls located at the triangle edge and vertex and above and below the ellipse, as shown in the PFM amplitude image in Fig. 5(a). A DC bias was then applied to move the domain walls in-situ. The PFM amplitude in Fig. 5(b) shows the resulting movement of the domain walls as the applied voltage increased from $11 \mathrm{~V}$ to $25 \mathrm{~V}$ in $1 \mathrm{~V}$ increments (incrementing every 5-10 scan lines, marked in Fig. 5). The final domain wall configuration after the switching experiment can be seen at the bottom of Fig. 5(b) (below the $0 \mathrm{~V}$ applied bias level). In this case, the relative displacements of the domain walls do appear to correlate qualitatively with the expected degree of local field enhancement.

\section{CONCLUSION}

In conclusion, we have found that patterning of both asperities on electrodes and air holes in the ferroelectric within the interelectrode gap can cause site-specific nucleation of reverse domains during switching from a fully poled state. Nucleation events can be separated in terms of the levels of applied bias at which they occur, thus giving the possibility of selecting different numbers of domain walls traversing the interelectrode gap, depending on the bias levels applied-a necessary development for the selection of specific resistance states in a domain wall-based memristor device. The nucleation sites associated with local field hotspots were successfully predicted by the FEA modeling although the expected sequence of nucleation events as a function of the increasing bias did not always correlate with the theoretical predictions (which might be an indication that further improvement in FEA modeling has to be introduced).

\section{ACKNOWLEDGMENTS}

The authors acknowledge financial support from the Materials World Network (MWN) scheme involving the Engineering and Physical Sciences Research Council in the UK (EP/H047093/1) and the National Science Foundation in the USA (DMR-1007943). International networking support from The Leverhulme Trust (F/00 203/ V) is also gratefully acknowledged. J.M.G. and R.G.P.M. acknowledge financial support from the Engineering and
Physical Sciences Research Council (EP/J017191/1). J.M.G. and J.R.W. acknowledge support from the Department of Employment and Learning (DEL).

${ }^{1}$ L. Chua, IEEE Trans. Circuit Theory 18, 507 (1971).

${ }^{2}$ D. B. Strukov, G. S. Snider, D. R. Stewart, and R. S. Williams, Nature 453, 80 (2008).

${ }^{3}$ J. J. Yang, M. D. Pickett, X. Li, D. A. Ohlberg, D. R. Stewart, and R. S. Williams, Nature Nanotech. 3, 429 (2008).

${ }^{4}$ N. D. Mathur, Nature 455, E13 (2008).

${ }^{5}$ A. Chanthbouala, V. Garcia, R. O. Cherifi, K. Bouzehouane, S. Fusil, X. Moya, S. Xavier, H. Yamada, C. Deranlot, N. D. Mathur et al., Nature Mater. 11, 860 (2012).

${ }^{6}$ S. H. Jo, T. Chang, I. Ebong, B. B. Bhadviya, P. Mazumder, and W. Lu, Nano Lett. 10, 1297 (2010).

${ }^{7}$ D. J. Kim, H. Lu, S. Ryu, C. W. Bark, C. B. Eom, E. Y. Tsymbal, and A. Gruverman, Nano Lett. 12, 5697 (2012).

${ }^{8}$ J. J. Yang, D. B. Strukov, and D. R. Stewart, Nature Nanotech. 8, 13 (2012).

${ }^{9}$ H. Yamada, V. Garcia, S. Fusil, S. Boyn, M. Marinova, A. Gloter, S. Xavier, J. Grollier, E. Jacquet, C. Carrétéro et al., ACS Nano 7, 5385 (2013).

${ }^{10}$ Y. Liu, X. Lou, M. Bibes, and B. Dkhil, Phys. Rev. B 88, 024106-1 (2013).

${ }^{11}$ D. Kim, H. Lu, S. Ryu, S. Lee, C. Bark, C. Eom, and A. Gruverman, Appl. Phys. Lett. 103, 142908 (2013).

${ }^{12}$ Z. Hu, Q. Li, M. Li, Q. Wang, Y. Zhu, X. Liu, X. Zhao, Y. Liu, and S. Dong, Appl. Phys. Lett. 102, 102901 (2013).

${ }^{13}$ Z. Wang, W. Zhao, W. Kang, A. Bouchenak-Khelladi, Y. Zhang, J.-O. Klein, D. Ravelosona, and C. Chappert, J. Appl. Phys. 114, 044108 (2013).

${ }^{14}$ Z. Wen, C. Li, D. Wu, A. Li, and N. Ming, Nature Mater. 12, 617 (2013).

${ }^{15}$ A. Aird and E. K. H. Salje, J. Phys.: Condens. Matter 10, L377 (1998).

${ }^{16}$ A. Aird, M. Domeneghetti, F. Mazzi, V. Tazzoli, and E. K. H. Salje, J. Phys.: Condens. Matter 10, L569 (1998).

${ }^{17}$ J. Seidel, L. W. Martin, Q. He, Q. Zhan, Y. H. Chu, A. Rother, M. Hawkridge, P. Maksymovych, P. Yu, M. Gajek et al., Nature Mater. 8, 229 (2009).

${ }^{18}$ E. K. H. Salje, Chem. Phys. Chem. 11, 940 (2010).

${ }^{19}$ J. Guyonnet, I. Gaponenko, S. Gariglio, and P. Paruch, Adv. Mater. 23, 5377 (2011).

${ }^{20}$ Y. Kaneko, Y. Nishitani, H. Tanaka, M. Ueda, Y. Kato, E. Tokumitsu, and E. Fujii, J. Appl. Phys. 110, 084106 (2011).

${ }^{21}$ Y. Du, X. Wang, D. Chen, S. Dou, Z. Cheng, M. Higgins, G. Wallace, and J. Wang, Appl. Phys. Lett. 99, 252107 (2011).

${ }^{22}$ S. Farokhipoor and B. Noheda, Phys. Rev. Lett. 107, 127601 (2011).

${ }^{23}$ P. Maksymovych, J. Seidel, Y. H. Chu, P. Wu, A. P. Baddorf, L. Q. Chen, S. V. Kalinin, and R. Ramesh, Nano Lett. 11, 1906 (2011).

${ }^{24}$ D. Meier, J. Seidel, A. Cano, K. Delaney, Y. Kumagai, M. Mostovoy, N. A. Spaldin, R. Ramesh, and M. Fiebig, Nature Mater. 11, 284 (2012).

${ }^{25}$ E. A. Eliseev, A. N. Morozovska, G. S. Svechnikov, P. Maksymovych, and S. V. Kalinin, Phys. Rev. B 85, 045312 (2012).

${ }^{26}$ A. N. Morozovska, R. K. Vasudevan, P. Maksymovych, S. V. Kalinin, and E. A. Eliseev, Phys. Rev. B 86, 085315 (2012).

${ }^{27}$ P. Maksymovych, A. Morozovska, P. Yu, E. Eliseev, Y. H. Chu, R. Ramesh, A. Baddorf, and S. Kalinin, Nano Lett. 12, 209 (2012).

${ }^{28}$ W. Wu, Y. Horibe, N. Lee, S. W. Cheong, and J. Guest, Phys. Rev. Lett. 108, 77203 (2012).

${ }^{29}$ S. Van Aert, S. Turner, R. Delville, D. Schryvers, G. V. Tendeloo, and E. K. H. Salje, Adv. Mater. 24, 523 (2012).

${ }^{30}$ Y. Kim, M. Alexe, and E. K. H. Salje, Appl. Phys. Lett. 96, 032904 (2010).

${ }^{31}$ A. Schilling, T. Adams, R. Bowman, and J. Gregg, Nanotechnology 18, 035301 (2007).

${ }^{32}$ V. Atuchin, N. Y. Maklakova, L. Pokrovsky, and V. Semenenko, Opt. Mater. 23, 363 (2003).

${ }^{33}$ J. R. Whyte, R. G. P. McQuaid, P. Sharma, C. Canalias, J. F. Scott, A. Gruverman, and J. M. Gregg, Adv. Mater. 26, 293 (2014). 